\title{
Quit smoking advice from health professionals in Taiwan: the role of funding policy and smoker socioeconomic status
}

\author{
Fong-ching Chang, ${ }^{1}$ Teh-wei Hu, ${ }^{2}$ Shu-ying Lo, ${ }^{3}$ Po-tswen Yu, ${ }^{3}$ Kun-yu Chao, ${ }^{3}$ \\ Mei-ling $\mathrm{Hsiao}^{3}$
}

${ }^{1}$ National Taiwan Normal University, Taipei, Taiwan ${ }^{2}$ University of California, Berkeley, USA ${ }^{3}$ Bureau of Health Promotion, Department of Health, Taiwan

\section{Correspondence to}

Fong-ching Chang, National Taiwan Normal University, Taiwan, 162, Ho-Ping E Road, Sec. 1, Taipei 10610, Taiwan; fongchingchang@ntnu.edu.tw

Received 13 May 2009 Accepted 28 October 2009 Published Online First

3 December 2009

\section{(2) UNLOKK:}

This paper is freely available online under the BMJ Journals unlocked scheme, see http:// tobaccocontrol.bmi.com/site/ about/unlocked.xhtml.

\begin{abstract}
Objectives In 2002, Taiwan launched a program to encourage doctors to provide brief cessation counselling to their patients during routine outpatient visits. This study is to compare and analyse the annual prevalence rate of receiving advice to quit smoking from health professionals before $(2004)$ and after $(2005,2006)$ the increase in funding and the withdrawal of additional funding (2007).
\end{abstract}

Methods We analysed pooled data from 2004 to 2007 Taiwan Adult Tobacco Survey, an annual random digit dialling telephone survey, to estimate the prevalence of receiving quit advice among ever smokers across these years. Smoking characteristics and the socioeconomic factors of smokers associated with receipt of advice to quit smoking were also examined.

Results The prevalence rate of receiving quit advice increased from $21.1 \%$ in 2004 to $28.2 \%$ in 2006 , and then decreased slightly to $27.6 \%$ in 2007 after the funds were cut. Multivariate analyses results indicated that increasing financing for smoking cessation services in 2005, being male, older, a daily cigarette user, having previously attempted to quit, perceiving oneself as having poor health and being aware of the benefits of smoking cessation services were significantly positively associated with receiving quit advice from health professionals. In contrast, smokers who were younger, female and occasional cigarette users were less likely to receive quit advice. Also, smokers with socioeconomic disadvantages were not less likely to receive quit advice. Conclusions During the period of increased funding for smoking cessation services, the rates of receiving quit advice increased among all smokers and across different socioeconomic groups.

\section{INTRODUCTION}

The World Health Organization (WHO) has predicted that tobacco may kill a billion people by the end of this century. More than three-quarters of tobacco-attributable deaths will be in low-income and middle-income countries. The WHO have emphasised that individual country healthcare systems hold primary responsibility to provide tobacco treatment. The provision of brief quit advice should be integrated into primary healthcare services and other routine medical visits. ${ }^{1}$ Some countries, such as the USA, ${ }^{2}$ the $\mathrm{UK}^{3}$ and Australia, ${ }^{4}$ have published evidence-based guidelines to recommend effective tobacco cessation interventions ranging from brief quit advice to extensive counselling combined with pharmaceutical adjuncts.
A meta-analysis found that a brief advice intervention can increase quitting by a further $1 \%$ to $3 \%{ }^{5}$ Another study found that offering basic quit advice could prevent about $13 \%$ of myocardial infarctions and $19 \%$ of strokes. ${ }^{6}$ Despite these guidelines and studies showing cost-effective ways of preserving life and reducing illness, health professionals in many countries underperform in helping smokers quit, ${ }^{7-12}$ and most smokers report not being offered cessation advice. ${ }^{13-16}$

Taiwan has a smoking prevalence of $22 \%$; $39 \%$ of men and $5 \%$ of women smoke. Cigarette smoking in Taiwan has resulted in an estimated 18000 deaths annually. Taiwan has about 4 million smokers, over half of whom have attempted to quit at least once. ${ }^{17}$ Taiwan launched National Health Insurance in 1995 to provide healthcare for all citizens. However, smoking cessation treatments are not covered under the insurance plan. Health professionals seldom provide smoking cessation advice to their patients. The country enacted a tobacco health and welfare tax in 2002, the same year that it implemented the Smoking Cessation Outpatient Services program designed to encourage doctors to integrate smoking cessation counselling in routine outpatient visits. Taiwan's smoking cessation counselling guidelines recommend a 6-min counselling agenda termed the 'five As': ask, advise, assess, assist and arrange. Contracted doctors are required to attend a 6 -h smoking cessation consultation/training workshop. Subjects are to receive a brief consultation about smoking cessation from health professionals (ie, doctors, nurses and pharmacists) and a prescription of medication that might help them quit smoking (eg, nicotine replacement therapy and bupropion).

The Smoking Cessation Outpatient Services program was designed to offer doctors financial incentives to provide brief cessation counselling during routine outpatient visits. An additional 250 New Taiwan dollars (NT\$, equivalent to US\$8) of doctor reimbursement was granted for delivering cessation counselling. However, only 20000 smokers $(0.5 \%$ of all smokers) had received treatment for their addiction in 2003. To increase the number of patients receiving smoking cessation services, the Taiwanese government increased reimbursement and medication subsidies starting in January 2005. Under the new funding policy, doctors started receiving NT\$350 (US\$11) per visit instead of NT\$250 (US\$8) they were receiving in 2004. Also, the government offered a subsidy of cessation medications to eligible subjects aged 19 
years or older who were smoking at least 10 cigarettes per day. In 2005 , subjects could receive medication subsidies of up to NT $\$ 400$ (US\$13) per week instead of NT\$250 (US\$8) per week before the increase, while smokers with low income could receive higher medication subsidies of up to NT $\$ 500$ (US\$16) per week. Under the new program, every patient could receive two courses (8 weeks per course) on smoking cessation each year. In addition, the eligibility criteria for participating doctors were expanded from family practice, psychiatry and internal medicine to all other specialties including gynaecology, otolaryngology and surgery.

After the increase in financial support and the broadening of medical specialisations that can offer cessation services in 2005 , the number of contracted doctors increased from 1841 (5.5\% of all doctors) in 2004 to 3466 (10.4\%) in 2005 and 4161 (11.9\%) in 2006. Annual number of cessation services delivered increased 5 times, from 22167 subjects (44554 visits) in 2004 to 109508 subjects (273754 visits) in $2005 .{ }^{18}$ However, as the number of smoking cessation services increased, total cost also increased from $\$ 0.8$ million in 2004 to $\$ 7$ million in 2005. Due to a budget shortage, in April 2006 the government reduced the funding to the pre-January 2005 levels. Enrolled doctors still receive an additional US\$8 for cessation counselling. Subjects also receive medication subsidies of US\$8 per week, while low-income people continue to receive higher medication subsidies (US\$16 per week). Reimbursement data show that the number of smokers receiving smoking cessation treatments decreased since April 2006. ${ }^{19}$ However, whether the changes in the funding policy for smoking cessation services influenced cessation advice rates in large, population-based samples of smokers is unclear.

Smoking is associated with socioeconomic disadvantage and is an important contributor to health inequalities. The socioeconomic characteristics, insurance coverage, ethnicity and health status of smokers have been shown to influence receipt of smoking cessation advice. ${ }^{15} 20-23$ In addition, the policy of funding changes for tobacco control programs is a critical factor in influencing the implementation and outcome of tobacco use prevention and cessation. ${ }^{18} 24-26$ Despite studies that have shown a positive impact of financial reimbursement and insurance coverage on the provision of cessation counselling and utilisation of smoking cessation services, ${ }^{18} 27-30 \mathrm{few}$ studies have evaluated the impact of changing funding for smoking cessation services on population-based cessation advice rates. This study aims to determine whether the changes in funding for smoking cessation services influenced trends in annual prevalence rates of receiving quit advice from health professionals during 2004-2007. We also assessed differences in the annual prevalence rates of receiving quit advice across these years by smoker socioeconomic status in Taiwan. Smoker characteristics related to receipt of quit advice also were examined.

\section{METHODS}

This study used national population-based data from the 2004, 2005, 2006 and 2007 Taiwan Adult Tobacco Survey to assess the prevalence of receiving quit advice from health professionals. The Taiwan Adult Tobacco Survey is an annual cross-sectional, county-based random dialling telephone survey to estimate smoking behaviour and attitudes in a representative sample of the non-institutionalised adult population (age 18 and older) in Taiwan. The survey is conducted by the Bureau of Health Promotion, Taiwan. Telephone numbers are drawn from 5 strata categorised by the population size of 25 counties. In each county, the sample number of successfully interviewed individuals ranged from 400 to 800 based on the different strata. Phone numbers were proportionally selected and called by random digit sampling. In each household, a representative adult sample was randomly selected. The household telephone coverage rate in Taiwan was up to $98 \%$ since 2000 . The response rates were about $60 \%$ for the 2004-2007 surveys.

In the 2004, 2005, 2006 and 2007 Taiwan Adult Tobacco Surveys, representative samples of 16688, 16749, 16922 and 16588 people were interviewed successfully by telephone. According to the 2004, 2005, 2006 and 2007 databases, a total of 5358, 4846, 5220 and 4866 adults, respectively, were ever smokers, while 3292, 3131, 3072 and 2953 adults were current smokers. Individuals were identified as ever smokers by the question "Have you smoked at least 5 packs (100 cigarettes) in your entire life?". Then individuals were asked "Do you now smoke cigarettes every day, some days, or not at all?". To determine smoking cessation advice by health professionals, ever smokers were asked: "During the past 12 months did any doctor, nurse, pharmacist, or other health professionals advise you to quit smoking?". Thus, all ever smokers were included in this study to assess the rates of receiving quit advice vis a vis different funding policies and smoker characteristics.

The independent variable in this study was receipt of quit advice from health professionals. Respondents who reported that they did not receive quit advice from health professionals were categorised as the reference group. The dependent variables included the funding policy for smoking cessation services and smoker characteristics. The different periods of funding policy for smoking cessation services were measured by year (2004 2005, 2006 and 2007). Since the Taiwan government increased the funding for smoking cessation services from January 2005 through March 2006, the year 2004 was categorised as the reference group. Smoker characteristics obtained in this study included smoking status (ever vs current), cigarette use (daily vs occasional), quit attempts in the past year (yes vs no), perceived health status (good vs poor), their awareness of smoking cessation services (yes vs no), gender (male vs female), age (18-34, 35-49, 50-64, 65+), household monthly income (NT\$, $\leq 20000, \quad 20001-60000$, 60001-100000, $\geq 100001$ ), educational attainment (elementary, middle, high school, college) and employment (yes vs no).

SAS software (V.9.1; SAS, Cary, North Carolina, USA) was used to analyse the data sets. To assess the prevalence of receiving quit advice in the 2004-2007 surveys, estimates were weighted to the respondent's probability of being selected and the age-specific, sex-specific and education-specific populations from the 2000 Taiwan census. Trend analyses were used to test the changes in the annual prevalence of receiving quit advice in 2004, 2005, 2006 and 2007. Univariate and multivariate logistic regression were conducted to examine the relationships between the rates of receipt of advice to quit smoking from health professionals and the funding policy for smoking cessation services as well as smoker characteristics.

\section{RESULTS}

\section{Smoking rates by demographic characteristics}

After weighting the survey design, the overall prevalence of smoking among adults in Taiwan in 2004, 2005, 2006 and 2007 was $23.9 \%, 22.2 \%, 21.4 \%$ and $21.1 \%$, respectively, while the prevalence of former smokers was $6.4 \%, 5.7 \%, 7.4 \%$ and $7.4 \%$. Current cigarette smoking prevalence was $42.9 \%$, 39.3\%, $38.4 \%$ and $37.6 \%$ for men in 2004, 2005, 2006 and 2007, respectively, while smoking prevalence for women was $4.5 \%, 4.7 \%, 4.1 \%$ and $4.7 \%$ (table 1). As age increased, the percentage of current smokers decreased. Adults with at least a bachelor's degree were 
Table 1 Prevalence rates of smoking by demographic characteristics

\begin{tabular}{|c|c|c|c|c|}
\hline & \multicolumn{4}{|c|}{ Weighted percentage (\%) } \\
\hline & 2004 & 2005 & 2006 & 2007 \\
\hline Total & 23.9 & 22.2 & 21.4 & 21.1 \\
\hline \multicolumn{5}{|l|}{ Gender } \\
\hline Female & 4.5 & 4.7 & 4.1 & 4.7 \\
\hline Male & 42.9 & 39.3 & 38.4 & 37.6 \\
\hline \multicolumn{5}{|l|}{ Age } \\
\hline $18-34$ & 24.4 & 21.4 & 20.3 & 19.7 \\
\hline $35-49$ & 26.8 & 26.2 & 24.5 & 26.1 \\
\hline $50-64$ & 21.1 & 20.1 & 19.8 & 18.9 \\
\hline $65+$ & 19.0 & 17.2 & 18.9 & 16.3 \\
\hline \multicolumn{5}{|l|}{ Education } \\
\hline Elementary & 18.3 & 18.8 & 18.2 & 16.4 \\
\hline Middle & 34.9 & 31.7 & 29.2 & 31.3 \\
\hline High school & 27.7 & 26.1 & 25.6 & 24.6 \\
\hline College and higher & 16.5 & 14.1 & 13.8 & 14.3 \\
\hline \multicolumn{5}{|c|}{ Household monthly income } \\
\hline NT $\$ \leq 20000$ & 21.1 & 19.1 & 18.8 & 19.7 \\
\hline NT\$20001-60000 & 26.1 & 25.3 & 22.7 & 22.5 \\
\hline NT\$60001-100000 & 26.5 & 23.9 & 23.2 & 22.8 \\
\hline NT $\$ \geq 100001$ & 30.8 & 29.7 & 26.1 & 26.1 \\
\hline Unknown & 21.4 & 18.5 & 19.4 & 17.6 \\
\hline \multicolumn{5}{|l|}{ Employment } \\
\hline No & 14.9 & 14.4 & 14.4 & 13.9 \\
\hline Yes & 30.3 & 28.3 & 26.6 & 26.5 \\
\hline
\end{tabular}

Using Taiwan year 2000 census as sample weights. $\mathrm{N}=16688$ (2004), 16749 (2005) 16922 (2006) and 16588 (2007).

NT\$, new Taiwan dollars.

less likely than other adults to be current smokers. For example, current smoking prevalence for those with college and higher education level was $16.5 \%, 14.1 \%, 13.8 \%$ and $14.3 \%$ in 2004 , 2005, 2006 and 2007, respectively, while smoking rate for those with middle school education level was $34.9 \%, 31.7 \%, 29.2 \%$ and $31.3 \%$. In addition, smoking rate among adults having household monthly income less than NT\$20000 was lower than the rate among adults having higher income. Overall, the mean age of smokers was 42 years; $81 \%$ of smokers were daily smokers; $74 \%$ had employment; $20 \%$ had a household monthly income less than NT $\$ 2000$; almost half of smokers had attempted to quit in the preceding year.

\section{Receipt of quit advice by different funding periods}

The increased funding for smoking cessation services in 2005 appears to have increased the prevalence rates of receiving quit advice during the previous year from $21.1 \%$ in 2004 to $26.8 \%$ in 2005 and $28.2 \%$ in 2006. However, the rate declined slightly to $27.6 \%$ in 2007 as the funding policy reverted to the pre-January 2005 levels in April 2006. By gender, the prevalence rate of receiving quit advice among male smokers increased from $21.9 \%$ in 2004 to $29.0 \%$ in 2006 and then slightly decreased to $28.7 \%$ in 2007, while the prevalence rate among female smokers increased from $14.7 \%$ in 2004 to $21.3 \%$ in 2006 and then decreased to $19.3 \%$ in 2007.

\section{Receipt of quit advice by smoker characteristics}

Table 2 presents the trends in receipt of quit advice during 2004-2007 by smoker characteristics. Trend analysis of the 2004-2007 data indicated an overall increase in the rates of receipt of quit advice across different smoker characteristic groups with the increased funding for smoking cessation services in 2005. However, the prevalence rates declined slightly in 2007 after the funds were cut. For example, for older smokers, the
Table 2 Prevalence rates of receiving quit advice from health professionals

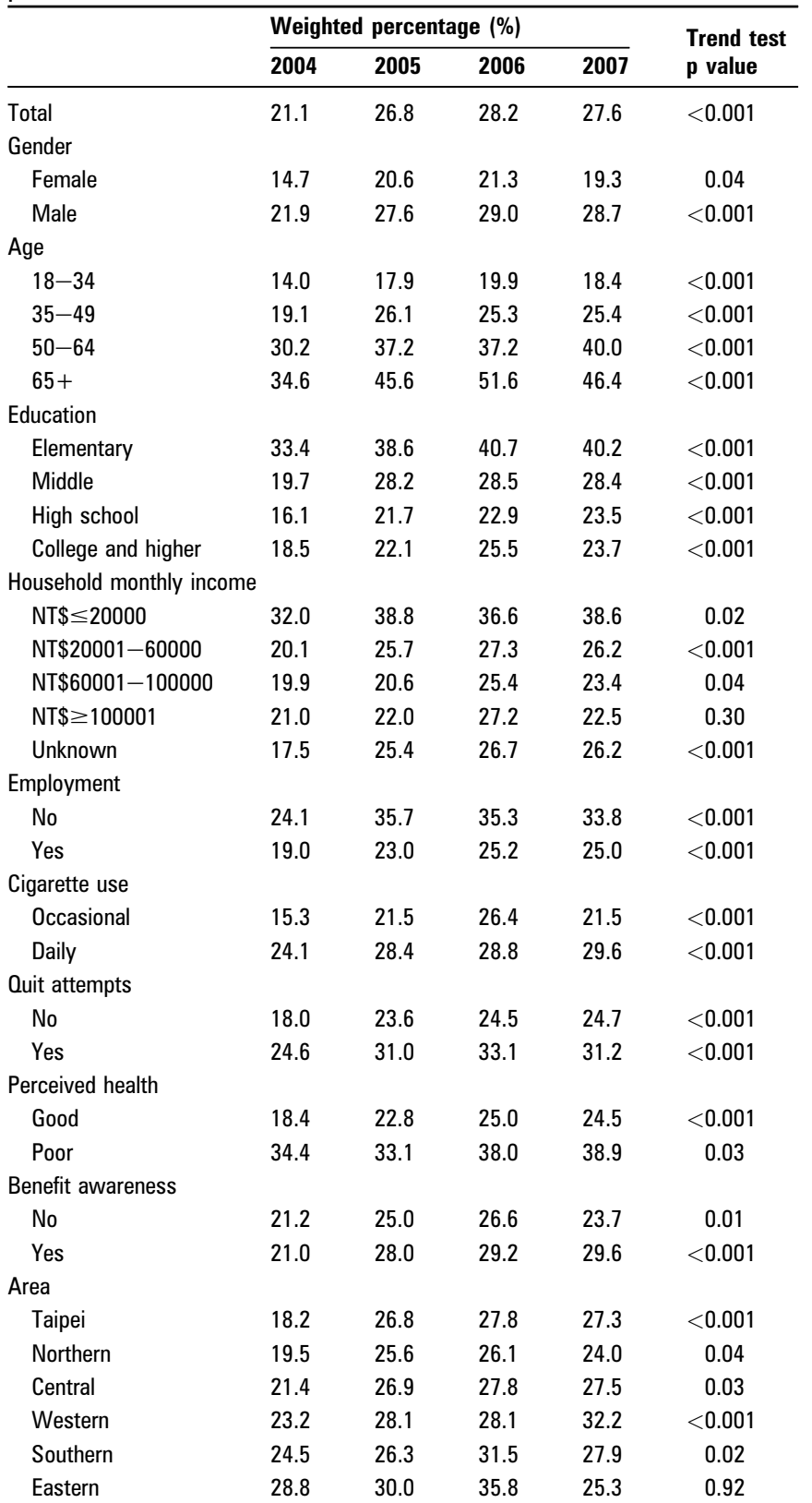

Note: 1. Using Taiwan year 2000 census as sample weights. $\mathrm{N}=5358$ (2004), 4846 (2005), 5220 (2006) and 4866 (2007).

NT\$, new Taiwan dollars.

prevalence rate of receiving quit advice increased from $34.6 \%$ in 2004 to $51.6 \%$ in 2006 and then decreased to $46.4 \%$ in 2007 . Similarly, for occasional cigarette users, the prevalence rate increased from $15.3 \%$ in 2004 to $26.4 \%$ in 2006 and then decreased to $21.5 \%$ in 2007. In addition, for smokers having household monthly income less than NT\$20 000, the prevalence rate of receiving quit advice increased from $32.0 \%$ in 2004 to $38.6 \%$ in 2007.

\section{Factors associated with receiving quit advice}

The univariate analysis results showed that increasing financing for smoking cessation services in 2005 was related to receiving quit advice. For example, the odds of receiving quit advice in the past 12 months in the 2006 survey was 1.40 times the odds in the 2004 survey (table 3 ). In addition, those who were male, older, less 
Table 3 Factors associated with receipt of smoking cessation services

\begin{tabular}{|c|c|c|c|c|}
\hline & \multicolumn{2}{|c|}{ Unadjusted } & \multicolumn{2}{|c|}{ Adjusted } \\
\hline & $\overline{\mathbf{O R}}$ & $95 \% \mathrm{Cl}$ & $\overline{\mathbf{O R}}$ & $95 \% \mathrm{Cl}$ \\
\hline \multicolumn{5}{|l|}{ Financing policy } \\
\hline 2004 & 1 & & 1 & \\
\hline 2005 & 1.23 & 0.91 to 1.64 & 1.26 & 1.11 to 1.42 \\
\hline 2006 & 1.40 & 1.05 to 1.85 & 1.39 & 1.25 to 1.56 \\
\hline 2007 & 1.34 & 1.00 to 1.77 & 1.37 & 1.22 to 1.53 \\
\hline \multicolumn{5}{|l|}{ Gender } \\
\hline Female & 1 & & 1 & \\
\hline Male & 1.74 & 1.19 to 2.53 & 1.50 & 1.29 to 1.74 \\
\hline \multicolumn{5}{|l|}{ Age } \\
\hline $18-34$ & 1 & & 1 & \\
\hline $35-49$ & 1.50 & 1.36 to 1.65 & 1.49 & 1.34 to 1.66 \\
\hline $50-64$ & 2.51 & 2.24 to 2.81 & 2.38 & 2.08 to 2.73 \\
\hline $65+$ & 3.35 & 2.96 to 3.79 & 3.29 & 2.75 to 3.93 \\
\hline \multicolumn{5}{|l|}{ Education } \\
\hline Elementary & 1 & & 1 & \\
\hline Middle & 0.61 & 0.54 to 0.69 & 0.86 & 0.74 to 1.01 \\
\hline High school & 0.46 & 0.41 to 0.51 & 0.72 & 0.62 to 0.83 \\
\hline College and higher & 0.48 & 0.43 to 0.54 & 0.77 & 0.67 to 0.90 \\
\hline \multicolumn{5}{|l|}{ Income } \\
\hline NT\$ $\leq 20000$ & 1 & & 1 & \\
\hline NT\$20001-60000 & 0.60 & 0.54 to 0.67 & 0.98 & 0.85 to 1.12 \\
\hline NT\$60001-100000 & 0.58 & 0.50 to 0.66 & 1.00 & 0.84 to 1.18 \\
\hline $\mathrm{NT} \$ \geq 100001$ & 0.56 & 0.48 to 0.65 & 1.02 & 0.85 to 1.23 \\
\hline Unknown & 0.57 & 0.51 to 0.64 & 0.98 & 0.85 to 1.13 \\
\hline \multicolumn{5}{|l|}{ Employment } \\
\hline No & 1 & & 1 & \\
\hline Yes & 0.69 & 0.64 to 0.75 & 1.04 & 0.93 to 1.17 \\
\hline \multicolumn{5}{|l|}{ Cigarette use } \\
\hline Occasional & 1 & & 1 & \\
\hline Daily & 1.63 & 1.50 to 1.78 & 1.74 & 1.58 to 1.92 \\
\hline \multicolumn{5}{|l|}{ Quit attempts } \\
\hline No & 1 & & 1 & \\
\hline Yes & 1.50 & 1.39 to 1.62 & 1.75 & 1.61 to 1.90 \\
\hline \multicolumn{5}{|l|}{ Perceived health } \\
\hline Good & 1 & & 1 & \\
\hline Poor & 2.12 & 1.92 to 2.34 & 2.03 & 1.82 to 2.25 \\
\hline \multicolumn{5}{|l|}{ Benefit awareness } \\
\hline No & 1 & & 1 & \\
\hline Yes & 1.15 & 1.06 to 1.24 & 1.29 & 1.18 to 1.41 \\
\hline \multicolumn{5}{|l|}{ Area } \\
\hline Taipei & 1 & & 1 & \\
\hline Northern & 0.92 & 0.82 to 1.05 & 0.92 & 0.81 to 1.06 \\
\hline Central & 1.01 & 0.89 to 1.13 & 0.97 & 0.85 to 1.10 \\
\hline Western & 1.12 & 1.00 to 1.26 & 1.03 & 0.91 to 1.17 \\
\hline Southern & 1.10 & 0.97 to 1.24 & 1.02 & 0.89 to 1.16 \\
\hline Eastern & 1.24 & 1.06 to 1.45 & 1.06 & 0.89 to 1.26 \\
\hline
\end{tabular}

Using univariate and multivariate logistic regression. In the multivariate logistic regression, $\mathrm{N}=13$ 192; received $\mathrm{n}=3293$; not received $\mathrm{n}=9899,-2 \log \mathrm{L}=13$ 789.5.

NT\$, new Taiwan dollars.

educated, daily cigarette users, who had lower income, had previously attempted to quit and who perceived themselves as having poor health were more likely to receive quit advice from health professionals. For example, the odds of receiving quit advice for men was 1.74 times that of women. Similarly, smokers who had attempted to quit in the past 12 months had 1.50 times the odds of receiving quit advice compared to smokers who had not previously attempted to quit. Smokers who perceived themselves as having poor health had 2.12 times the odds of receiving quit advice compared to smokers who perceived themselves as having good health. Moreover, increased age had a strong effect on receiving cessation advice. For instance, compared to smokers between 18 and 34 years of age, the odds of receiving quit advice for smokers over 65 years old was the highest (OR 3.35), followed by smokers between 50 and 64 years old (OR 2.51), followed by those between 35 and 49 years old (OR 1.50).

Results of the multivariate analyses indicated that increasing financing for smoking cessation services in 2005, being male, older, a daily cigarette user, having previously attempted to quit, perceiving oneself as having poor health, and being aware of the benefits of smoking cessation services were significantly positively associated with receiving quit advice. After adjusting for other variables, the odds of receiving quit advice (OR 1.26) increased after increasing funding for smoking cessation services in 2005. In 2006, the odds of receiving quit advice continuously increased (OR 1.39). In addition, after adjusting for other variables, smokers who were aware of the benefits of financing smoking cessation services had 1.29 times the odds of receiving quit advice than smokers not aware of the benefits. Similarly, after adjusting for other variables, receiving smoking cessation advice remained significantly higher for older individuals, males (OR 1.50), daily cigarette users (OR 1.74), those who had attempted to quit in the past year (OR 1.74) and those with perceived poor health (OR 2.03). However, after adjusting for other variables, employment and income status variables were no longer significantly associated with receiving smoking cessation advice.

\section{DISCUSSION}

Increased financing for smoking cessation services has been positively related to provider involvement in consultation and smoker utilisation of smoking cessation services. ${ }^{18} 2627$ This study provides additional evidence to indicate that financing smoking cessation services appears to improve the rates of population-based receipt of quit advice. The results showed that the prevalence rates of receiving quit advice increased from $21.1 \%$ in 2004 (prior to the funding increase in 2005) to $28.2 \%$ in 2006. Then the rate declined slightly to $27.6 \%$ in 2007 as the funding policy reverted to the pre-January 2005 levels in April 2006. However, the decrease was not significant (OR 0.94, 95\% CI 0.84 to 1.05). After increased funding for smoking cessation service, more doctors (12\% of all doctors) from different specialisations participated in this program. In addition, local governments encouraged doctors in all township health centres to deliver smoking cessation services to enhance accessibility. Moreover, several programs such as smoking cessation media campaigns, community smoking cessation projects, health professionals smoking cessation training program, smoking cessation services for hospitalised patients, and a pilot study of the development of smoker identification system in public hospitals may also boost effects on receipt of quit advice from health professionals.

Nevertheless, the probability of not being offered advice in Taiwan $(71 \%)$ remained high compared to the rates in the UK $(59 \%)^{13}$ and the US (39\%). ${ }^{31}$ Studies ${ }^{8}{ }^{32-35}$ have identified barriers to the low rates of health professional advice to quit smoking: time constraints, lack of financial incentives, lack of training and materials, perceived low effectiveness and being smokers. In Taiwan, $70 \%$ of the tobacco health tax was allocated to the National Health Insurance Security Fund. However, smoking cessation treatments are not covered under the National Health Insurance Plan. A doctor often sees more than 50 patients in a morning or an afternoon. Most doctors seldom ask about patients' smoking status and provide smoking cessation advice. The government should restructure the healthcare financing and accreditation systems to facilitate the institutionalisation of systems changes and adopt tobacco cessation interventions as an integral element of healthcare delivery. 
Prior studies ${ }^{15} 20$ found a complex relationship between socioeconomic status and receipt of quit advice, and the results were mixed. Our study found that the increased funding policy was associated with increasing prevalence of smoking cessation advice among smokers with socioeconomic disadvantages. The receipt rate of quit advice was higher for smokers with a household monthly income of less than NT\$20000 than for other smokers. The univariate results showed that smokers with low education or low income had a higher probability of receiving quit advice. However, after adjusting for other factors, the socioeconomic factor was unrelated to the receipt of quit advice. This may be due to a strong negative correlation between household income level and age. Our results showed that smokers with household monthly income less than NT\$20000 were mainly people over 65 years old (56\%) and those between 50 and 64 years old $(22.3 \%)$.

Under the Taiwan National Health Insurance system, lowincome people are eligible to get full insurance coverage, while other residents have to pay a copayment. The implementation of a copayment system is to prevent people from seeking medical care that may not be necessary. However, the copayment mechanism may also discourage people, especially for lowincome group, from seeking necessary medical care. To reduce financial barrier for low-income people to attend medical services, the government use the tobacco tax revenue to subside low-income people's premium. Also, low-income smokers could receive higher subsidies for cessation medication starting in 2005. Since many healthcare sectors in Taiwan provide drugs to patients, additional reimbursement for cessation counselling and medication may increase incentives for providers, especially for clinics, to deliver cessation services. As indicated above, the current policy of increasing financial incentives for providers and accessibility for low-income people may subsequently reduce health disparities by facilitating quit attempts for smokers with socioeconomic disadvantages.

Our findings are generally in accord with previous studies $^{13} 203637$ that found smokers who had chronic diseases and were older were more likely to receive quit advice from providers. In contrast to some other studies, our study found that female smokers, occasional cigarette users and younger smokers were less likely to be given advice. Some prior studies ${ }^{13} 20$ found being a female smoker increased the probability of receiving quit advice. In Taiwan, the female smoking rate is $5 \% .{ }^{17}$ Perhaps providers think that women are less likely to smoke, and it is thus less necessary to provide quit advice. Providers also may not easily recognise occasional cigarette users compared to daily users. The implementation of a smoker identification system should be promoted in all healthcare sectors to facilitate health professionals routinely giving quit advice and assisting patients to quit smoking. ${ }^{38}$ Gender-sensitive smoking cessation programs should be developed to better target female smokers.

Our study also found that the smoking rate among younger people was higher. However, younger smokers and smokers with employment were less likely to report having received assistance to quit. In contrast, smokers who had attempted to quit and were aware of the benefits of smoking cessation were more likely to receive quit advice. Tobacco dependence is recognised as a chronic disease that requires ongoing repeated interventions and multiple attempts to quit. ${ }^{2}$ Outpatient visits per person per year average over 14 in Taiwan. ${ }^{39}$ One study found that receipt of quit advice by multiple health professionals increased quit attempts and quitting. ${ }^{40}$ If a large number of health professionals offer cessation advice, then the cumulative effect on reducing smoking rate and disease burden could be substantial. ${ }^{41}$ Moreover, worksite smoking cessation programs should be promoted to increase the accessibility of cessation services for young adults. Population-wide strategies, such as smoke-free environments, increasing the tobacco tax and smoking cessation campaigns should continue to expand to create the demand for smoking cessation services.

This study has some limitations. First is the potential threat to validity from such factors as historical events. For example, smoking cessation media campaigns during the study period may have influenced the receipt rates of quit advice. Second, this study used existing data sets that did not collect information concerning organisational and healthcare provider characteristics, which might influence the delivery of cessation advice. Third, over a quarter of the respondents did not report their income. The missing data were placed in the 'unknown cases' group and included in the analysis. Fourth, data were based on the self-report of smokers, who may not have remembered smoking cessation assistance they received over a 12-month period.

Finally, another challenge to estimate the prevalence of receiving quit advice is to decide who to include in the denominator. Previous studies often used current smokers in the denominator, but that approach may lose some smokers who received cessation advice and then quit during the preceding year. The US guideline suggests that clinicians should provide every patient who uses tobacco with at least a brief intervention targeted and effective for smokers willing to quit, smokers unwilling to quit and former tobacco users. ${ }^{2}$ This study used ever smokers in the denominator. Analyses showed quit advice prevalence among ever smokers was slightly lower than that of current smokers. For example, the rate of receiving quit advice among ever smokers in 2004, 2005, 2006 and 2007 was $21.1 \%$, $26.8 \%, 28.2 \%$ and $27.6 \%$, respectively, while the rate among current smokers was $24.1 \%, 27.7 \%, 28.2 \%$ and $28.2 \%$. Since the smoking prevalence in Taiwan decreased from 23.9\% in 2004 to $21.1 \%$ in 2007, the prevalence of receiving quit advice among ever smokers in 2007 may have been less. In addition, 28\% of ever smokers answered 'not sure/unknown' with respect to receiving quit advice. Moreover, ever smokers who answered 'not sure/ unknown' tended to have a higher percentage (37\%) in the age group over 50 years compared to that of smokers who did not (26\%).

In conclusion, trends analyses of the 2004-2007 data indicated that the prevalence of receiving quit advice increased across different socioeconomic groups with the appearance of increased funding for smoking cessation services, while the prevalence declined slightly after the funds were cut. Among smokers in Taiwan, older men, who were daily cigarette users, had attempted to quit in the past year, had perceived poor health and were aware of the benefits of smoking cessation services were more likely to report ever having received advice on quitting from health professionals, while female, younger and occasional cigarette users were less likely to have received quit advice. Nevertheless, smokers with socioeconomic disadvantages were not less likely to receive quit advice. Quitting advice is an important process for the purpose of smoking cessation. Research should further examine who had successfully quit smoking and environmental influences such as the healthcare organisation policy on provider delivery of cessation advice, smokers' receiving quit advice and smoking cessation rates at the population level.

Acknowledgements We thank Professor Steven Schroeder and two anonymous reviewers for their helpful comments and suggestions.

Competing interests None. 


\section{What this paper adds}

The increased funding for smoking cessation services appears to have improved the prevalence rate of receiving quit advice from health professionals.

- The rates for receipt of quit advice across different socioeconomic groups increased during the increased funding period.

- Smokers who were younger, female and occasional cigarette users were less likely to receive quit advice from health professionals, while smokers with socioeconomic disadvantages were not less likely to receive quit advice.

Ethics approval The study was approved by the Bureau of Health Promotion, Department of Health, Taiwan and the Office for the Protection of Human Subjects at UC Berkeley.

Provenance and peer review Not commissioned; externally peer reviewed.

\section{REFERENCES}

1. World Health Organization. MPOWER: a policy package to reverse the tobacco epidemic Geneva: World Health Organization, 2008.

2. Fiore MC. A clinical practice guideline for treating tobacco use and dependence: 2008 update. A U.S. Public Health Service report. Am J Prev Med 2008;35:158-76.

3. West R, McNeill A, Raw M. Smoking cessation guidelines for health professionals: an update. Health Education Authority. Thorax 2000;55:987-99.

4. Zwar N, Richmond R, Borland R, et al. Smoking cessation guidelines for Australian general practice. Aust Fam Physician 2005;34:461-6.

5. Stead LF, Bergson G, Lancaster T. Physician advice for smoking cessation. Cochrane Database Syst Rev 2008(2):CD000165.

6. Eddy DM, Peskin B, Shcheprov A, et al. Effect of smoking cessation advice on cardiovascular disease. Am J Med Qual 2009;24:241-9.

7. Hu S, Pallonen U, McAlister AL, et al. Knowing how to help tobacco users. Dentists' familiarity and compliance with the clinical practice guideline. J Am Dent Assoc 2006;137:170-9.

8. Pipe A, Sorensen M, Reid R. Physician smoking status, attitudes toward smoking, and cessation advice to patients: an international survey. Patient Educ Couns 2009:74:118-23.

9. Yee C, Gansky SA, Ellison JA, et al. Tobacco control in pediatric dental practices: a survey of practitioners. Pediatr Dent 2008:30:475-9.

10. Jiang Y, Ong MK, Tong EK, et al. Chinese physicians sand their smoking knowledge, attitudes, and practices. Am J Prev Med 2007;33:15-22.

11. Thorndike AN, Regan S, Rigotti NA. The treatment of smoking by US physicians during ambulatory visits: 1994 2003. Am J Public Health 2007;97:1878-83.

12. Raw M, Regan S, Rigotti NA, et al. A survey of tobacco dependence treatment services in 36 countries. Addiction 2009:104:279-87.

13. Twigg L, Moon G, Szatkowski L, et al. Smoking cessation in England: intentionality, anticipated ease of quitting and advice provision. Soc Sci Med 2009:68:610-19.

14. Ferketich AK, Gallus S, Colombo P, et al. Physician-delivered advice to quit smoking among Italian smokers. Am J Prev Med 2008;35:60-3.

15. Browning KK, Ferketich AK, Salsberry PJ, et al. Socioeconomic disparity in providerdelivered assistance to quit smoking UK: Nicotine \& Tobacco Research, Oxford University Press, 2008.

16. Stevenson J, Snider J, Kaiserman MJ. Smoking-cessation advice from health-care providers - Canada, 2005. MMWR Morb Mortal Wkly Rep 2007;56:708-12.

17. Bureau of Health Promotion. Taiwan Tobacco Control 2008 anual report. Taipei (Taiwan): Bureau of Health Promotion, Department of Health, 2008.

18. Chang FC, Hu TW, Lin M, et al. Effects of financing smoking cessation outpatient services in Taiwan. Tob Control 2008;17:183-9.
19. Office of Taiwan Smoking Cessation Services. Taiwan smoking cessation oupatient services 2007 annual report Taipei (Taiwan): Bureau of Health Promotion, Department of Health, 2008.

20. Houston TK, Scarinci IC, Person SD, et al. Patient smoking cessation advice by health care providers: the role of ethnicity, socioeconomic status, and health. Am J Public Health 2005:95:1056-61.

21. Curry SJ, Sporer AK, Pugach 0, et al. Use of tobacco cessation treatments among young adult smokers: 2005 National Health Interview Survey. Am J Public Health 2007:97:1464-9.

22. Lopez-Quintero C, Crum RM, Neumark YD. Racial/ethnic disparities in report of physician-provided smoking cessation advice: analysis of the 2000 National Health Interview Survey. Am J Public Health 2006;96:2235-9.

23. Reed MB, Burns DM. A population-based examination of racial and ethnic differences in receiving physicians' advice to quit smoking. Nicotine Tob Res 2008: 10:1487-94.

24. Niederdeppe J, Farrelly MC, Hersey JC, et al. Consequences of dramatic reductions in state tobacco control funds: Florida, 1998-2000. Tob Control 2008;17:205-210.

25. Jones AS, Austin WD, Beach RH, et al. Funding of North Carolina tobacco control programs through the Master Settlement Agreement. Am J Public Health 2007:97:36-44.

26. Coleman T, Lewis S, Hubbard R, et al. Impact of contractual financial incentives on the ascertainment and management of smoking in primary care. Addiction 2007; 102:803-8

27. Reda AA, Kaper J, Fikrelter $\mathrm{H}$, et al. Healthcare financing systems for increasing the use of tobacco dependence treatment. Cochrane Database Syst Rev 2009(2): CD004305.

28. Roski J, Jeddeloh $\mathrm{R}, \mathrm{An} \mathrm{L}$, et al. The impact of financial incentives and a patient registry on preventive care quality: increasing provider adherence to evidence-based smoking cessation practice guidelines. Prev Med 2003;36:291-9.

29. Kaper J, Wagena EJ, Willemsen MC, et al. Reimbursement for smoking cessation treatment may double the abstinence rate: results of a randomized trial. Addiction 2005; 100:1012-20.

30. Curry SJ, Grothaus LC, McAfee T, et al. Use and cost Effectiveness of smokingcessation services under four insurance plans in a Health Maintenance Organization. N Engl J Med 1998;339:673-9.

31. Cokkinides VE, Halpern MT, Barbeau EM, et al. Racial and ethnic disparities in smoking-cessation interventions: analysis of the 2005 National Health Interview Survey. Am J Prev Med 2008:34:404-412.

32. Helgason AR, Lund KE. General practitioners' perceived barriers to smoking cessation-results from four Nordic countries. Scand J Public Health 2002;30:141-7

33. Vogt F, Hall S, Marteau TM. General practitioners' and family physicians' negative beliefs and attitudes towards discussing smoking cessation with patients: a systematic review. Addiction 2005;100:1423-31.

34. Twardella D, Brenner $\mathrm{H}$. Lack of training as a central barrier to the promotion of smoking cessation: a survey among general practitioners in Germany. Eur J Public Health 2005;15:140-5.

35. Squier C, Hesli V, Lowe J, et al. Tobacco use, cessation advice to patients and attitudes to tobacco control among physicians in Ukraine. Eur J Cancer Prev 2006;15:458-63.

36. Ossip-Klein DJ, Mclntosh S, Utman C, et al. Smokers ages 50+: who gets physician advice to quit? Prev Med 2000;31:364-9.

37. Lucan SC, Katz DL. Factors associated with smoking cessation counselling at clinica encounters: the Behavioral Risk Factor Surveillance System (BRFSS) 2000. Am J Health Promot 2006:21:16-23.

38. Fiore MC, Keller PA, Curry SJ. Health system changes to facilitate the delivery of tobacco-dependence treatment. Am J Prev Med 2007;33(Suppl 6):S349-356.

39. Bureau of National Health Insurance. Introduction of Taiwan Nation Health Insurance Services, 2009. Taipei (Taiwan): Bureau of National Health Insurance, Department of Health, 2009.

40. An LC, Foldes SS, Alesci NL, et al. The impact of smoking-cessation intervention by multiple health professionals. Am J Prev Med 2008;34:54-60.

41. National Cancer Institute. Population based smoking cessation: proceedings of a conference on what works to influence cessation in the general population Bethesda (MD): U.S. Department of Health and Human Services, National Institutes of Health National Cancer Institute, 2000. 